\title{
Observation of Depictive Versus Tracing Gestures Selectively Aids Verbal Versus Visual-Spatial Learning in Primary School Children
}

\author{
MARGOT VAN WERMESKERKEN ${ }^{1,2} *$, NATHALIE FIJAN ${ }^{1}$, CHARLY EIELTS $^{1}$ and \\ WIM T. J. L. POUW ${ }^{1}$ \\ ${ }^{1}$ Department of Psychology, Education, and Child Studies, Erasmus University Rotterdam, Rotterdam, The Netherlands \\ ${ }^{2}$ Department of Education, Utrecht University, Utrecht, The Netherlands
}

\begin{abstract}
Summary: Previous research has established that gesture observation aids learning in children. The current study examined whether observation of gestures (i.e. depictive and tracing gestures) differentially affected verbal and visual-spatial retention when learning a route and its street names. Specifically, we explored whether children $(n=97)$ with lower visual and verbal workingmemory capacity benefited more from observing gestures as compared with children who score higher on these traits. To this end, 11- to 13-year-old children were presented with an instructional video of a route containing no gestures, depictive gestures, tracing gestures or both depictive and tracing gestures. Results indicated that the type of observed gesture affected performance: Observing tracing gestures or both tracing and depictive gestures increased performance on route retention, while observing depictive gestures or both depictive and tracing gestures increased performance on street name retention. These effects were not differentially affected by working-memory capacity. Copyright (C) 2016 John Wiley \& Sons, Ltd.
\end{abstract}

\section{INTRODUCTION}

Smooth and efficient human communication involves hand gesticulation (e.g. Özyürek, 2014; Streeck et al., 2011). Teaching can be regarded as a special kind of communication as the teacher has to be attuned to the cognitive dispositions of the learner and the learning content involved (e.g. Kline, 2014). Perhaps, then, hand gesticulation in teaching should also be attuned to the cognitive dispositions of the learner (e.g. working-memory capacity) and the learning content involved (e.g. verbal vs. visual-spatial content). This is the topic of the present study.

\section{Gesture and learning: Different gesture different effects?}

It is now well established that observing gestures can positively affect learners' performance (for a recent overview, see Novack \& Goldin-Meadow, 2015; see also Alibali \& Nathan, 2012). For example, children (5-7 years) learning about quantity conservation (i.e. Piagetian conservation task) improve more when the instructor gestures as compared with when the instructor does not gesture (Ping \& GoldinMeadow, 2008). In a similar vein, supplementing verbal instruction with meaningful gestures during a lesson about mathematical equivalence can boost retention and transfer performance in children (7-10 years); notably this effect was observed 24 hours after the lesson (Cook et al., 2013). In addition, positive effects of gesture observation have been found in, for example, learning about symmetry (Valenzeno et al., 2003), lightning formation (De Koning \& Tabbers, 2013) and novel verb learning (Macedonia et al., 2011).

Hence, the types of tasks wherein gesture observation can support learning are quite diverse. If we consider that gesticulation comes in many forms (Streeck et al., 2011), it is likely that observing particular types of gestures is especially effective for particular types of cognitive feats. Previous research has already

*Correspondence to: Margot van Wermeskerken, Department of Education, Utrecht University, P.O. Box 80140, 3508 TC Utrecht, The Netherlands. E-mail: m.m.vanwermeskerken@uu.nl been engaged with this topic in several ways (e.g. Alibali \& Nathan, 2012; Austin \& Sweller, 2014; Novack et al., 2014). For example, children (8-11 years) observing and then reproducing and applying abstract symbolic gestures (e.g. v-shape gesture) during mathematical equivalence learning seems to boost performance as opposed to more action-like gestures (e.g. grasping gesture) or physical interactions with the learning materials (e.g. manipulation of objects, Novack et al., 2014; see however, Fischer et al., 2015). More relevant for the present study, it has been found that children (3-4 years) can benefit from observing specific gestures in encoding verbally presented route directions (Austin \& Sweller, 2014). In this study, next to the effect of depictive gesture (i.e. gestures that physically enact or depict the meaning of speech) observation, the researchers were interested in beat gestures. Beat gestures are rhythmic movements of the hand that may help to accentuate or highlight speech segments. Indeed, it was found that children (but not adults) improved in recall of route directions when these were communicated with beat gestures alone, or depictive and beat gestures, as compared with no gestures.

Two types of gestures that are likely to have very different cognitive functions for observers are tracing (which can be considered deictic gestures) and depictive gestures (also called representational gestures). It has been argued that depictive gestures provide additional non-categorical information, which provides opportunities for deeper or more contextualized processing of meaning (Alibali \& Nathan, 2012). Indeed, it has been found that such information is picked up by observers, wherein the gesture observed is often reproduced in the observers' own gestures performed moments afterward (Cook \& Tanenhaus, 2009; see also Austin \& Sweller, 2014). In contrast, tracing gestures are held to provide observers with a way to spatially index or anchor speech in the environment. For example, it has been found that pre-school children's (4-5 years) learning about symmetry increased when instructional videos included a model performing tracing gestures as opposed to an instructional video with only verbal instruction. Given the clear contrast between depictive and tracing gestures, we aim to investigate whether the observation of such gestures indeed 
differentially affects verbal and visual components when learning a single navigational task and to examine whether these effects are affected by learners' cognitive dispositions (i.e. visual-spatial and verbal working-memory capacity).

\section{Gesture and cognitive dispositions}

If observing depictive and tracing gestures is cognitively potent for learning particular learning content, they may also be especially potent for those learners who have the most difficulty with that particular learning content. For example, if verbal skills are compromised, the learner may need extra help in the form of depictive gestures. Or, when visual-spatial skills are lacking, tracing gestures may be a welcome addition to instruction.

Indications that this might be the case come from research and theory on gesture production rather than observation.

This line of research has been more engaged with the role of cognitive dispositions in gesture effects (for an overview, see Pouw et al., 2014). For example, depictive gesturing, and also tracing gesturing, has been found to counteract workingmemory load (Cook et al., 2013; Goldin-Meadow et al., 2001; Ping \& Goldin-Meadow, 2008; Smithson \& Nicoladis, 2014; Wagner et al., 2004). That is, participants who were allowed (vs. restricted) to gesture during explaining a solution of a math or physics problem remembered more items of the secondary task (e.g. 2-d spatial coordinates or strings of letters), which suggests that gesturing lowered the working memory load imposed by the primary (i.e. explanation) task (Goldin-Meadow et al., 2001; Ping \& Goldin-Meadow, 2008; Wagner et al., 2004). Furthermore, when using pointing and tracing gestures (as opposed to no gestures), those problem solvers who have a lower verbal workingmemory capacity were more likely to benefit from gesturing in recalling strings of letters after explaining a math task (Marstaller \& Buríanová, 2013).

On the basis of this line of research, it has been argued that the production of gestures is valuable because they allow a way to alleviate internal cognitive processes (Pouw et al., 2014; Pouw \& Hostetter, in press). That is, producing gestures provides stable proprioceptive and visual feedback that can be used to maintain or bring forth task-relevant information. For example, it has been shown that gesturing — as if manipulating objects - during mental rotations aids performance (Chu \& Kita, 2011). It has been suggested that these gestures are effective for mental rotation as they allow to perform a rotation in external space, which can be mapped onto internal mental rotations. In effect, the gesture is argued to reduce the complexity of the task by distributing load over brain and body and thereby alleviating internal cognitive load (Pouw et al., 2014).

In the present study, it is explored whether this line of reasoning, that is, the rationale of gesture production as a compensation mechanism for managing limited internal cognitive resources, can be directly applied to gesture observation. Specifically, gesture observation may alleviate the observers' need to internally process communicative content, as part of this content is already expressed externally by the speaker in his or her gestures. Congenial to the predictions made by Pouw et al. (2014) concerning the effect of gesture production, it is possible that gesture observation is more effective for those who have more difficulty with processing speech without external aids, that is, have lower working memory load. By contrast, learners who are more cognitively disposed are supposed to benefit to a lesser extent from observing gestures, as they have less difficulty with processing speech and are thus less in need of such external aids (but see De Nooijer et al., 2013; Wu \& Coulson, 2014).

Our line of reasoning involves the risky assumption (given the sparse research on this matter) that observing gestures affects cognitive performance in a similar way as producing gestures. That is, if research on producing depictive gestures is correct (e.g. Cook et al., 2012), depictive gestures allow to alleviate verbal working memory load (e.g. remembering strings of letters as a secondary task) by distributing the semantic content of some communicative message (e.g. selfexplanation of a math problem) over the manual and speech modality rather than the speech modality alone. Given our rationale, it would follow that observing depictive gestures (next to content in speech) too would alleviate verbal processing load in the observer, as semantic content is conveyed not only in the speech modality (requiring verbal processing) but also the visual-spatial modality. In contrast, if research on the production of tracing gestures is correct (e.g. So et al., 2014; So et al., 2015), such gestures are supporting visual-spatial processing (e.g. route learning) and can be effective even when no verbal content is involved (co-thought tracing; So et al., 2015). As such, we deem it more likely that observing tracing gestures is effective because they target visuo-spatial rather than verbal processing. Yet, we hasten to note that our prediction of the differential working memory support of depictive versus tracing gestures is about differences in degree of association, rather than assuming that depictive or tracing gestures is only related to either verbal or visual-spatial processing. Finally, we note that the choice for these specific working-memory capacity components is motivated by findings that verbal and visual working-memory capacity is predictive for the rate at which participants produce co-speech gestures (Chu et al., 2013; Gillespie et al., 2014; see also Pouw et al., 2016), providing some preliminary evidence (if our rationale is on track) that such working memory components might relate to gesture observation as well.

In sum, even though previous research has already established beneficial effects of observing gestures during learning, its effectiveness in relation to the learners' cognitive disposition is still ill understood and understudied, especially in research with children. However, combined with the assumption that particular gestures are effective for conveying particular types of information, it seems likely that observation of particular types of gestures (e.g. tracing vs. depictive) are effective for performance of a particular component of a task (e.g. spatial vs. verbal), and especially for those who are less cognitively disposed (e.g. visual-spatial vs. verbal working-memory capacity) to solve that particular component of the task.

\section{Present study}

In the present study, we presented primary school children (1113 years) with a navigation retention task involving an instructional video with a human model. We investigated whether type of gesture observation during instruction (tracing and/or depictive gestures) selectively affected verbal (street name retention) or visual-spatial retention (route retention). 
Furthermore, we explored whether an effect of tracing and/or depictive gesture observation was selectively moderated by cognitive dispositions of the learner, namely, visual versus verbal working-memory capacity, which were assessed by the Visual Patterns Task (VPT; Della Sala et al., 1997) and Digit Span Task (DST; Wechsler, 1939). We predicted that tracing gestures are effective for visual-spatial retention (i.e. route retention) and explored whether this would be especially beneficial for those with a lower visual working-memory capacity. In addition, we predicted that depictive gestures are effective for verbal retention (i.e. street name retention) and explored whether this was especially beneficial for those with a lower verbal workingmemory capacity. If our prediction of the differential supportive function of gesture is correct, this would be expressed by working-memory capacity (verbal and/or visual) affecting the effectiveness of gesture observation in relation to task performance. However, note that, given the sparse literature on gesture observation in relation to working memory processes of the observer, the present hypotheses concerning working memory interactions should be regarded as exploratory in nature. In a similar vein, the combined gestures condition was included exploratory in order to assess whether seeing both gesture types might yield larger benefits for visual-spatial and verbal retention.

\section{METHODS}

\section{Participants}

Participants were 97 children (51 boys, $M_{\text {age }}=11.94$ years, $S D=0.48$ ) in their fifth or sixth year of primary school. All children participated based on parental consent, and the experiment was conducted in accordance with the institute's ethical guidelines. Children came from five different schools in the vicinity of Rotterdam and Amsterdam in the Netherlands. Forty-one additional children participated in the study but had to be excluded owing to technical issues (i.e. the online program intermittently stopped working; $n=25$ ) or owing to children not complying with the instructions $(n=16)$.

\section{Design}

Children were pseudo-randomly assigned to one of four conditions: the tracing condition (i.e. the model made tracing gestures to show the route on the map), the depictive condition (i.e. the model made depictive gestures to depict the street names), the depictive + tracing condition (i.e. the model made both tracing gestures to show the route on the map and depictive gestures to depict the street names) or the no gesture condition. Pseudo-randomization was carried out to assure that no differences between conditions would be the result of differences in intellectual ability of the children. Hence, children were pseudo-randomly assigned to one of four conditions based on their scores on Dutch national standardized sub-test scores (Cito Test) on comprehensive reading (Dutch: Cito Begrijpend Lezen) and mathematics (Dutch: Cito Rekenen/Wiskunde). These sub-test scores have been shown to correlate with verbal and visual-spatial working memory, respectively (e.g. Bull et al., 2008; Van der Ven et al., 2013; for an overview on the different cognitive competences related to verbal and visual- spatial working memory, see Baddeley, 2003). The Cito Test was administered 2 to 4 months prior to the current experiment. All scores were obtained from the participating schools prior to conducting the experiment. Cito scores were ranked on a scale from 1 to 5 based on the five-letter scale provided by Cito (based on national standardized scores: $1=25 \%$ best performing, $2=25 \%$ above average, $3=25 \%$ just below average, $4=15 \%$ below average, and $5=10 \%$ worst performing). Pseudo-randomization was performed by dividing children from one class into four groups based on their Cito scores, resulting in four groups that had approximately the same mean Cito score and spread. In doing so, the number of children of each level of mathematics and comprehensive reading ability was divided equally across the four groups. These groups were then randomly assigned to one of the four conditions. This resulted in four groups that were comparable with respect to their Cito sub-test scores (Table 1).

\section{Materials}

The entire series of tasks were programmed in Adobe Flash CC and JavaScript and administered on a computer using Qualtrics, an online survey program (www.qualtrics.com). Instructions for each task were given on-screen as text. Children could continue to the next screen by clicking a ' $>>$ ' button in the bottom right corner, after they had completed the tasks.

\section{Visual Patterns Task}

The VPT (adapted from Della Sala et al., 1997) measures visual working-memory capacity. The task was digitized and consisted of a series of grid patterns, wherein half of the squares were in black. Each trial consisted of a new pattern, which was displayed to the participant for 3 seconds, after which all the squares turned white (Figure 1). Children were then asked to recall the pattern of black squares by clicking the corresponding cells, which then turned black after they clicked it (by clicking a cell a second time, children could undo their action). The task consisted of 25 trials divided over five blocks of five trials, which increased in difficulty (i.e. going from seven to 13 black squares). To make children familiar with the task, two practice trials were presented (two and three black squares, respectively). In the case that children made two consecutive mistakes during these practice trials, the experimenter intervened and, when necessary, explained the task to the child before he or she continued. The task ended after 25 trials or when a participant made five consecutive mistakes within the same difficulty level. The test score was the proportion of correct trials (out of 25).

Table 1. Mean (and $S D$ ) of Cito sub-test scores presented for each condition

\begin{tabular}{lcc}
\hline & \multicolumn{2}{c}{ Cito sub-test scores } \\
\cline { 2 - 3 } & Comprehensive reading & Mathematics \\
\hline Control $(n=26)$ & $2.69(1.52)$ & $2.84(1.63)$ \\
Tracing $(n=23)$ & $2.61(1.62)$ & $2.59(1.47)$ \\
Depictive $(n=25)$ & $2.52(1.53)$ & $2.80(1.47)$ \\
Both $(n=23)$ & $2.43(1.47)$ & $2.70(1.55)$ \\
\hline
\end{tabular}

Note: Two children (one in the control condition and one in the tracing condition) were missing scores on the Cito test for mathematics.

$S D$, standard deviation. 

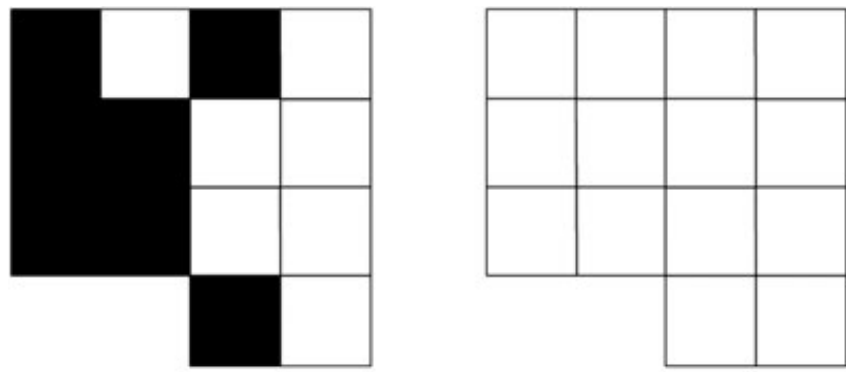

volgende

Figure 1. An example of the Visual Patterns Task. Left: the grid, which displays a pattern that had to be recalled; right: the same grid, but now the pattern had to be recalled by clicking on the corresponding squares

\section{Digit Span Task}

The DST measures verbal working-memory capacity (adapted from Wechsler, 1939). This task consisted of sequences of digits, presented at the rate of one digit per second. After each sequence, children had to recall the sequence in order of appearance and enter the digits by using the mouse (Figure 2). The task consisted of 25 trials that were presented in blocks of five trials, with each block going up in difficulty (i.e. five to nine digit sequences). The task was preceded by two practice trials to make children familiar with the task. In the case that children made two consecutive errors on the practice trials, the experimenter intervened and explained the task to the child before he or she continued. The task ended after 25 trials or when a participant made five consecutive mistakes within the same difficulty level. The test score was the proportion correct trials (out of 25).

\section{Example video}

In order to make children familiar with the subsequent map task, we presented an example video prior to the route video (see subsequent section). The video was made using Microsoft PowerPoint and contained animations to clarify the task and the steps that had to be performed. More specifically, in the video, a simple route (i.e. containing three steps) was explained verbally (no model visible). Subsequently, it was shown how the route was highlighted in the map, how the street name was entered and how the street was localized. The entire video lasted 62 seconds.

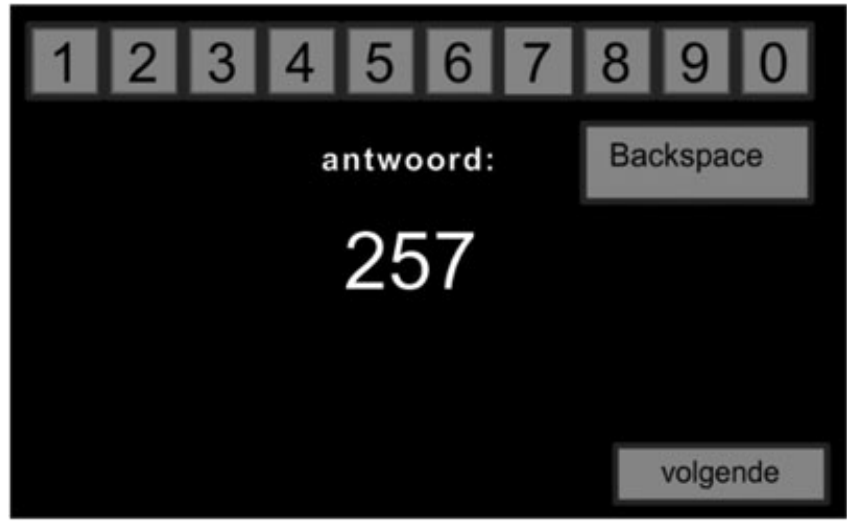

Figure 2. An example of the digitized Digit Span Task in which a sequence of numbers had to be recalled by clicking on the corresponding numbers

\section{Route video}

Four videos were constructed for each of the conditions. Each video featured a female model on the right, and a map on the left side of the screen (Figure 3). A pre-recorded female voice explained the route while, depending on the condition, the model produced or did not produce depictive, and/or tracing gestures. Because the directions were pre-recorded, the narration was exactly the same in each condition. In each video, the model's gaze was directed at the screen for the entire duration of the video.

The route that was explained in the video and that had to be recalled consisted of 12 directions (i.e. turning points) and four street names. The route started at a green dot and ended on the red dot, both of which were displayed on the map. All directions were given from an allocentric point of view (i.e. based on geographic directions: north, south, east and west). Street names were easily depictable using depictive gestures, such as 'Hammer Street' (Dutch: 'Hamerstraat'). In the tracing condition, tracing gestures were produced by the model by tracing at the map and following the given directions with her finger. In the depictive gestures condition, depictive gestures were produced by the model in order to depict the meaning of the street names, such as the motion of using a hammer in the case of the 'Hammer Street'. In the depictive + tracing condition, the model produced both types of gestures, that is, she pointed at the map and followed the directions, but she also performed depictive gestures to depict the meaning of the street names. In the control group, no gestures were used. A transcript of the directions is provided in Appendix A, and an image of the route is provided in Figure 4. Images and descriptions of the provided depictive gestures are included in Appendix B.

\section{Filler task}

After having studied the map task video and before conducting the test (refer to subsequent discussion), children performed a filler task. This was performed to prevent any recency effect and to ensure that the children stay engaged with the tasks without getting bored. This filler task consisted of a puzzle problem (i.e. frog leap) and lasted 2 minutes after which children proceeded to the test.

\section{Route test}

The route test consisted of three measures: retention of the route, retention of the street names, and their locations. Performance on

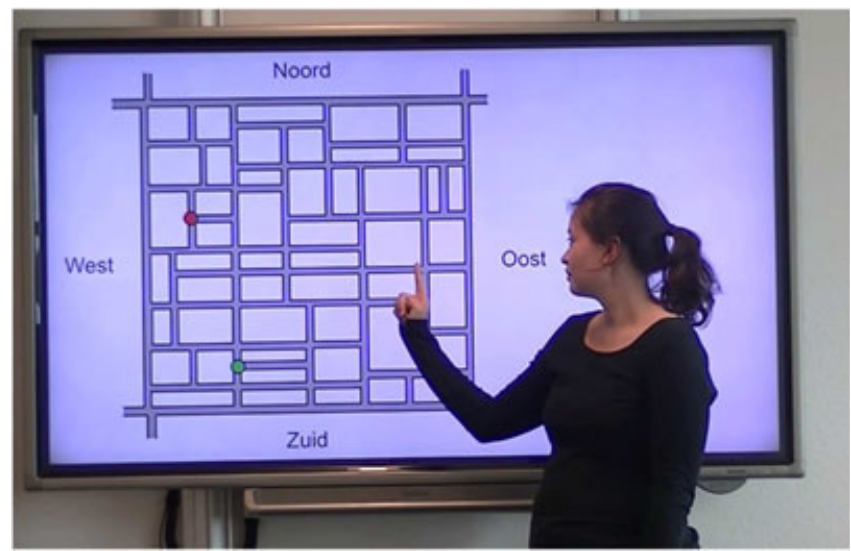

Figure 3. A screenshot of the video of the condition with tracing gestures only, showing the model and the map used in all conditions 


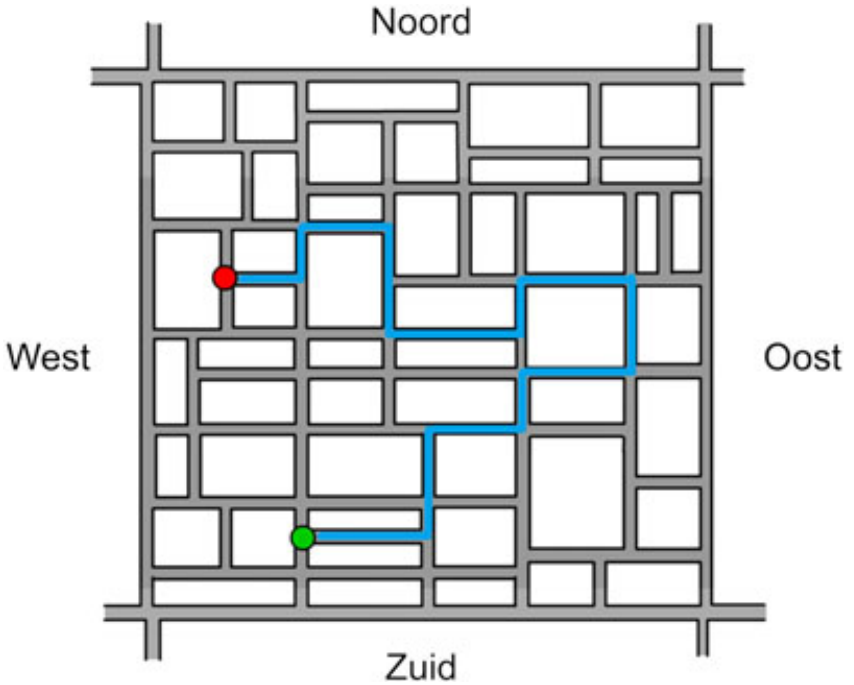

Figure 4 . The route that had to be recalled by the children is highlighted and consisted of steps (and 15 route segments), starting at the green dot and finishing on the red dot

each of these outcomes was measured using an interactive version of the map that was shown in the route video. Children had to indicate the route they remembered by clicking on and thereby highlighting the roads (by clicking a second time, children could undo their selection). Street names could be filled out at the designated blank typing spaces by using the keyboard, and markers could be dragged to the correct location of the particular street. An image of the final retention test screen is shown in Figure 4 (note that although children received 12 directions, they had to select 15 segments, as some of the directions contained information like 'take the second street to ...'; Appendix A).

\section{Procedure}

Children were tested in groups of four to 16 children, depending on the capacity of the computer room, with one or two experimenters. ${ }^{1}$ Before each testing session, the experimenter set up all computers, to make sure that children could start directly. Upon arrival of the children, the experimenter gave a brief explanation of what the children were about to do, without giving details about the tasks. Children were instructed to work quietly and to raise their hand in case of any questions or problems. Subsequently, children were asked to fill out some demographic questions (i.e. age, gender, and whether they were or were not dyslexic) and then were allowed to work on the tasks. Children first completed the working memory tasks, which were counterbalanced across conditions and children to avoid order effects. Therefore, half of the children in each condition first performed the DST and then the VPT; the other half started with the VPT and then performed the DST. On average, each working memory task took 8 minutes to complete. After these tasks, children were presented with the example video before the

\footnotetext{
${ }^{1}$ Note that participants were randomly assigned to gesture conditions. Hence, across conditions, group size was equal [one-way analysis of variance (ANOVA): $p=.511]$. Nevertheless, we computed Pearson correlations between the dependent variables (i.e. route retention and street name retention) and group size to assure that performance did not differ as a function of group size in which the children were tested. Correlations were not significant (street name retention, $r=.192, p=.060$; route retention, $r=.131$, $p=.200)$.
}

route video was presented. The children were specifically instructed to put their hands in their lap, to prevent them from gesturing while watching the video, which might affect learning (So et al., 2014). After the route video, children performed the filler task for 2 minutes. Finally, children performed the test that consisted of retention of the route, street names and location. Once the children had finished, they were asked to stay seated until all their peers had finished as well. They could then quietly return to their classrooms. Each testing session lasted 25 to 35 minutes.

\section{Data analysis}

\section{Dependent variables}

For both working memory tasks (VPT and DST), proportion of trials correct was determined out of a possible 25 .

For the route test, three components were scored: route retention, street name retention and street location. With respect to route retention, performance was determined based on the total number of correct route sections identified (out of 15) and correcting for the number of route sections that were incorrectly recalled (out of 114), according to this formula:

$$
\begin{aligned}
\text { Performance }= & \frac{\# \text { correct route section }}{15} \\
& -\frac{\# \text { incorrect route section }}{114}
\end{aligned}
$$

As such, the performance score could theoretically vary between -1 (in the case that a child had identified all but the correct route sections, which was never the case) and 1 (in the case that a child had identified all correct route sections and had not identified an incorrect route section).

With respect to street name retention, the proportion of correctly recalled street names was determined (out of four). Note that spelling errors did not affect street name retention scores.

Street name locations were counted as correct or incorrect based on in-task coordinates. Coordinates were saved by Adobe Flash and were then manually compared with a range of coordinates corresponding to the correct area on the map. The proportion of streets that were localized correctly was then determined (out of four).

\section{Statistical analyses}

First, Pearson correlations were computed between the Cito scores for mathematics and comprehensive reading and the working memory tasks, to investigate whether these were related to one another.

Second, we assessed gesture effects on the performance on each of the components of the route test separately using one-way ANOVAs with gesture conditions (i.e. tracing gestures and depictive gestures) as independent variables and performance on the route retention, street name retention and street name location as dependent variable. In order to assess the isolated effects of gesture type, we included both gesture types as independent variables with two levels: tracing gestures (yes/no) and depictive gestures (yes/no). In a second analysis, working memory scores were included as covariates in order to assess whether these variables affected the effects of the gesture conditions. 


\section{RESULTS}

All analyses used a significance level of .05. Cohen's $d$ and partial eta-squared are reported as a measure of effect size, with $d=0.20$ and $\eta_{\mathrm{p}}^{2}=0.02, d=0.50$ and $\eta_{\mathrm{p}}^{2}=0.13$, and $d=0.80$ and $\eta_{\mathrm{p}}^{2}=0.26$ corresponding to small, medium and large effects, respectively (Cohen, 1988).

An overview of the scores on both working memory tasks and performance on the route and street name retention tasks is provided in Table 2. Note that because performance on the street location task was extremely poor $(81.4 \%$ of the children did not select a single correct location or selected no location at all) and selection of the correct location depended highly on the correct selection of road sections, this variable was not included in the analyses. As can be observed in Table 2, performance on the DST was quite poor as well $(M=0.15, S D=0.11)$. Possible consequences of this finding will be discussed in the Discussion.

In a first step, we investigated whether there were any differences between conditions with respect to working memory scores (i.e. VPT and DST) using separate one-way ANOVAs. This did not reveal any significant differences between conditions (both $p s \geq .336$ ). Subsequently, in order to assess whether the used working memory tasks correlated with the Cito test scores on comprehensive reading and mathematics, we computed Pearson correlation coefficients between the working memory scores and the Cito test scores. Given that the VPT is assumed to measure visual working-memory capacity, which was related to the Cito test score on mathematics (Van der Ven et al., 2013), we expected a reliable correlation between the two scores. Indeed, it was revealed that the score on the VPT correlated significantly with the Cito test score on mathematics $(r=-.338, p=.001)$, but a slightly higher absolute correlation was apparent between the score on the VPT and the Cito test score on comprehensive reading $(r=-.372, p<.001)$. For the DST, which is a measure of verbal working-memory capacity, the opposite was expected, that is, a reliable correlation between the DST score and the Cito test score on comprehensive reading. Indeed, the DST score correlated significantly with the Cito test score on comprehensive reading $(r=-.265$, $p=.014)$, but again a slightly higher absolute correlation was revealed with the Cito test score on mathematics $(r=-.404$, $p<.001)$. Note, however, the correlations are all negative, which is in the opposite direction as anticipated and suggests that high performance on the working memory tasks is associated with low Cito scores and vice versa. This unexpected finding will be discussed in the Discussion.

\section{Gesture effects on route task performance}

In order to explore whether the different types of gestures differentially affected performance on the route test (i.e. route retention and street name retention), we performed two separate univariate 2 (depictive gestures: yes $/$ no) $\times 2$ (tracing gestures: yes/no) ANOVAs with the street retention and street name retention as dependent variables.

With respect to route retention, a significant effect was revealed for tracing gestures, $F(1,93)=32.12, p<.001, \quad \eta_{\mathrm{p}}^{2}$ $=0.26$, which indicated that route retention improved when tracing gestures were provided. The effect of depictive gestures was not significant, $F(1,93)=3.46, p=.066, \eta_{\mathrm{p}}^{2}=0.04$, and neither was the interaction effect of both gesture types, $F<1$. As we were interested in whether the combined gestures condition would outperform the single gesture and no gesture conditions, we conducted post-hoc comparisons (Bonferroni-adjusted $\alpha=.017$ due to three comparisons). These comparisons revealed that the combined gestures condition outperformed the no gestures condition $[t(47)=5.49, p<.001, d=1.60]$ and the depictive $[t(46)=3.87, p<.001, d=1.14]$, but not the tracing gestures condition $[t(44)=1.19, p=.241, d=0.36]$.

Finally, in order to assess whether route retention was affected by working-memory capacity scores (VPT and DST), we repeated this analysis with working memory scores included as covariate. This analysis did not reveal significant effects of the covariates; VPT, $F(1,80)=1.92, p=.170, \eta_{\mathrm{p}}^{2}=0.02$; DST, $F<1$. All other effects remained the same [tracing gestures, $F$ $(1,80)=29.37, p<.001, \eta_{\mathrm{p}}^{2}=0.27$; depictive gestures, $F(1$, $80)=3.89, p=.052, \eta_{\mathrm{p}}^{2}=0.05$; interaction, $\left.F<1\right]$.

For the proportion of correctly recalled street names, a significant effect of depictive gestures was observed, $F(1,93)$ $=42.383, p<.001, \eta_{\mathrm{p}}^{2}=0.31$, which indicated that more street names were recalled when depictive gestures were provided. Neither a main effect of tracing gestures nor an interaction between both gesture types was revealed (both $F \mathrm{~s}<1$ ). Again, we ran post-hoc comparisons (Bonferroni-adjusted $\alpha=.017$ due to three comparisons) in order to assess whether the combined gestures condition outperformed the single and no gestures condition. These comparisons revealed that the combined gestures condition outperformed the no gestures condition $[t(47)=5.20, p<.001, d=1.52]$ and the tracing $[t$ $(44)=4.62, p<.001, d=1.39$ ], but not the depictive gestures condition $[t(46)=1.43, p=.161, d=0.042]$.

In order to assess whether street name retention was affected by working memory scores (VPT and DST), we repeated this analysis with working memory scores included as covariates. This analysis did not reveal significant effects of the covariates; VPT, $F(1,80)=1.35, p=.249, \eta_{\mathrm{p}}^{2}=0.02$; DST, $F<1$. All other effects remained the same [depictive gestures, $F(1,80)=51.44$, $p<.001, \eta_{\mathrm{p}}^{2}=0.39$; tracing gestures, $F(1,80)=2.25, p=.137, \eta_{\mathrm{p}}^{2}$ $=0.03$; interaction, $F<1]$.

Table 2. Mean performance (and $S D$ ) on both working memory tasks and the route tasks presented for each condition

\begin{tabular}{|c|c|c|c|c|}
\hline & \multicolumn{2}{|c|}{ Working memory tasks } & \multicolumn{2}{|c|}{ Route tasks } \\
\hline & VPT & DST & Route retention & Street name retention \\
\hline Control & $0.50(0.19)$ & $0.17(0.10)$ & $0.28(0.19)$ & $0.37(0.28)$ \\
\hline Tracing & $0.49(0.17)$ & $0.12(0.11)$ & $0.51(0.21)$ & $0.36(0.34)$ \\
\hline Depictive & $0.51(0.16)$ & $0.13(0.11)$ & $0.36(0.21)$ & $0.68(0.21)$ \\
\hline Tracing + Depictive & $0.57(0.19)$ & $0.17(0.10)$ & $0.59(0.21)$ & $0.78(0.29)$ \\
\hline
\end{tabular}

Note: SD, standard deviation; VPT, Visual Patterns Task; DST, Digit Span Task. 


\section{DISCUSSION}

We investigated whether observing no gestures, tracing and depictive gestures, or a combination of both gesture types are effective for particular types of tasks (route retention vs. streetname retention). Furthermore, we assessed the exploratory hypotheses whether the gesture effect was more pronounced for those who were selectively disposed to have difficulty with a particular type of task. That is, we explored whether those with a lower visual-spatial working-memory capacity (as assessed by the DST) would benefit most from observing tracing gestures in performing of the street retention task (as compared with no gesture or depictive gesture observation). Additionally, we explored whether that those with a lower verbal working-memory capacity (as assessed by the VPT) would benefit most from depictive gestures on the street name retention task (as compared with no gesture or tracing gesture observation). Although our hypotheses about the differential effects of type of gesture on task performance were confirmed, our exploratory hypotheses that these effects might be moderated by working-memory capacity were not supported.

First of all, as predicted, strong effects (partial $\eta^{2}$ range: .26-.39; Cohen, 1988) of the type of observed gesture on performance were shown for both the route retention task and the street name retention task. More specifically, observation of tracing gestures or a combination of both tracing and depictive gestures improved recall of the route as compared with the no gesture or depictive gesture condition. In contrast, observation of depictive gestures or a combination of both tracing and depictive gestures selectively improved recall of street names as compared with the no gesture or depictive gesture condition. Yet, exploratory analyses revealed that for recall of the route, the combined gestures condition did not outperform the tracing gesture condition, suggesting that it was mainly the latter gestures that drove the effect. With respect to street name retention, the combination of depictive and tracing gestures did not yield better performance than the depictive gestures only condition.

These results suggest that indeed particular types of gestures are effective for particular types of tasks. We think that depictive gestures were especially effective for listeners' retention of street names as they solicit additional and deeper processing of the semantic content of a word (e.g. Alibali \& Nathan, 2012). That is, gesturing about the act of hammering provides a concrete context in which to understand the meaning of the word 'hammer' present in the street name 'Hammer Street'. Thus, we assume that providing a concrete context through depictive gesturing serves to give an additional multimodal meaning to a word, which in turn may also lead to a 'deeper' encoding and therefore greater retention. As indicated by other findings (e.g. Austin \& Sweller, 2014), gestural aids for deeper encoding might be especially important for children as opposed to adults, as they are still developing their verbal skills. In addition, we found that tracing gestures are effective for spatial retention. This effect can be explained in that tracing gestures are a natural sign (learned early in childhood; Gómez, 2007) that directs learners' attention in space. Externally directing attention on the map was in the present case especially helpful as the verbal instruction of the route had to be continuously spatially indexed on the map if not to lose track. It is further interesting to note that the present results indicate that children were able to reap information from tracing and depictive gestures in combination. This suggests that children 11-13 years are already very skilled gesture interpreters and can benefit from different types of gestures in close alternation, which convey meaning in quite different ways (e.g. tracing gestures are referential, while depictive gestures have more intrinsic meaning).

Yet, although the selective effects of type of gesture observation indicate that they provide the observer with particular cognitive benefits, those benefits were not found to be greater for those with constraints in relevant cognitive dispositions. That is, contrary to our exploratory predictions, no effects were found of visual-spatial versus verbal workingmemory capacity scores and the type of observed gesture on performance on the route tasks. This implies that observing specific kinds of gestures did not have an alleviating effect for those with lower working-memory capacities or for those with higher working-memory capacities.

Possible explanations for the finding that gestures did not alleviate working memory load for those who were less cognitively disposed (i.e. had lower working-memory capacity) might be twofold. First, it might be that the verbal working memory task (i.e. the DST) was too difficult, as was apparent by the overall low performance and little variance amongst participants. As such, this verbal working memory task might not have been valid and adequate in measuring children's verbal working memory especially considering the fact that the task is based on a test intended for adults (Wechsler, 1939). Future research therefore should take this into account by administering working memory tasks that are specifically targeted to and validated for this age group (e.g. Van de Weijer-Bergsma et al., 2014; Van de Weijer-Bergsma et al., 2015).

Second, and more fundamentally, it might be argued that the working memory tasks we chose might not have lined up directly with the kind of working memory needed to complete the route tasks (i.e. route retention and street name retention) successfully. This is indicated by the finding that performance on the route retention and street name retention tasks was not affected by the working memory scores. Hence, it seems that the verbal and visual-spatial working memory tasks did not relate with the cognitive strains imposed by the route retention and street retention task. This implies, for instance, that even though the working memory task we used has been shown to be a good measurement of visual-spatial working-memory capacity (e.g. Baddeley, 2003), the use of a navigational learning task possibly required a more spatial rather than visual working memory component (Coluccia et al., 2007; Garden et al., 2002). Further research using different measures for working-memory capacity should determine whether or not these effects exist.

Another surprising finding was that we did not find positive, but rather negative, correlations between the working-memory capacity scores and the Cito scores for comprehensive reading and mathematics. This observation is in contrast with previous research that established positive relationships between these measures (e.g. Bull et al., 2008; Van der Ven et al., 2013). As we used the Cito scores to make sure the four conditions were comparable with respect to cognitive dispositions, which was the case, this does not jeopardize the current findings. However, future research might shed more light onto the relationship between working memory measures, reading comprehension 
and mathematical ability and standardized sub-test scores, such as the Dutch Cito scores.

\section{CONCLUSION}

The current study showed that observation of particular types of gestures (i.e. depictive and tracing gestures) aids particular types of cognitive tasks (i.e. verbal and visualspatial, respectively). As such, these findings extend the large body of literature concerning the merits of gesture observation and gesture production (e.g. Novack \& GoldinMeadow, 2015) in showing that observation of different types of gestures differentially affects learning outcomes in children aged 11 to 13 years.

These findings may be of particular interest for applications in education. For instance, research has already shown that teachers can actively change the way they gesture to improve learning effectivity (e.g. Alibali et al., 2013; Hostetter et al., 2006). The present results can thus be applied in educational lessons, which combine spatial and verbal content, using different types of gestures (in combination) to benefit young observers in particular domains of learning.

\section{ACKNOWLEDGEMENTS}

This research was co-funded by the Netherlands Organisation for Scientific Research (NWO-PROO, project number 411-10-908). A special thanks to Monica Buurmeester for being our video model and to Astrid de Jong for being the second experimenter at one of the testing sessions.

\section{REFERENCES}

Alibali, M. W., \& Nathan, M. J. (2012). Embodiment in mathematics teaching and learning: Evidence from learners' and teachers' gestures. Journal of the Learning Sciences, 21, 247-286. DOI:10.1080/10508406.2011.611446.

Alibali, M. W., Young, A. G., Crooks, N. M., Yeo, A., Wolfgram, M. S., Ledesma, I. M., ... Knuth, E. J. (2013). Students learn more when their teacher has learned to gesture effectively. Gesture, 13, 210-233. DOI:10.1075/gest.13.2.05ali.

Austin, E. E., \& Sweller, N. (2014). Presentation and production: The role of gesture in spatial communication. Journal of Experimental Child Psychology, 122, 92-103. DOI:10.1016/j.jecp.2013.12.008.

Baddeley, A. (2003). Working memory: Looking back and looking forward. Nature Reviews Neuroscience, 4, 829-839. DOI:10.1038/nrn1201.

Bull, R., Espy, K. A., \& Wiebe, S. A. (2008). Short-term memory, working memory, and executive functioning in preschoolers: Longitudinal predictors of mathematical achievement at age 7 years. Developmental Neuropsychology, 33, 205-228. DOI:10.1080/87565640801982312.

Chu, M., \& Kita, S. (2011). The nature of gestures' beneficial role in spatial problem solving. Journal of Experimental Psychology: General, 140, 102-116. DOI:10.1037/a0021790.

Chu, M., Meyer, A., Foulkes, L., \& Kita, S. (2013). Individual differences in frequency and salience of speech-accompanying gestures: The role of cognitive abilities and empathy. Journal of Experimental Psychology: General, 143, 694-709. DOI:10.1037/a0033861.

Cohen, J. (1988). Statistical power analysis for the behavioral sciences (2nd edn). Hillsdale, NJ: Erlbaum.

Coluccia, E., Bosco, A., \& Brandimonte, M. A. (2007). The role of visuospatial working memory in map learning: New findings from a map drawing paradigm. Psychological Research, 71, 359-372. DOI:10.1007/ s00426-006-0090-2.
Cook, S. W., Duffy, R. G., \& Fenn, K. M. (2013). Consolidation and transfer of learning after observing hand gesture. Child Development, 84, 1863-1871. DOI:10.1111/cdev.12097.

Cook, S. W., \& Tanenhaus, M. K. (2009). Embodied communication: Speakers' gestures affect listeners' actions. Cognition, 113, 98-104. DOI:10.1016/j.cognition.2009.06.006.

Cook, S. W., Yip, T. K., \& Goldin-Meadow, S. (2012). Gestures, but not meaningless movements, lighten working memory load when explaining math. Language and Cognitive Processes, 27, 594-610. DOI:10.1080/ 01690965.2011.567074.

De Koning, B. B., \& Tabbers, H. K. (2013). Gestures in instructional animations: A helping hand to understanding non-human movements? Applied Cognitive Psychology, 27, 683-689. DOI:10.1002/acp.2937.

De Nooijer, J. A., Van Gog, T., Paas, F., \& Zwaan, R. A. (2013). Effects of imitating gestures during encoding or during retrieval of novel verbs on children's test performance. Acta Psychologica, 144, 173-179. DOI:10.1016/j.actpsy.2013.05.013.

Della Sala, S., Gray, C., Baddeley, A., \& Wilson, L. (1997). The Visual Patterns Test: A new test of short-term visual recall. Bury St Edmunds, England: Thames Valley Test.

Fischer, M. H., Sixtus, E., \& Göbel, S. M. (2015). Commentary: A pointer about grasping numbers. Frontiers in Psychology, 6, 227. DOI:10.3389/ fpsyg.2015.00227.

Garden, S., Cornoldi, C., \& Logie, R. H. (2002). Visuo-spatial working memory in navigation. Applied Cognitive Psychology, 16, 35-50. DOI:10.1002/acp.746.

Gillespie, M., James, A. N., Federmeier, K. D., \& Watson, D. G. (2014). Verbal working memory predicts co-speech gesture: Evidence from individual differences. Cognition, 132(2), 174-180. DOI:10.1016/j. cognition.2014.03.012.

Goldin-Meadow, S., Nusbaum, H., Kelly, S. D., \& Wagner, S. (2001). Explaining math: Gesturing lightens the load. Psychological Science, 12, 516-522. DOI:10.1111/1467-9280.00395.

Gómez, J. C. (2007). Pointing behaviors in apes and human infants: a balanced interpretation. Child Development, 78, 729-734. DOI:10.1111/ j.1467-8624.2007.01027.x.

Hostetter, A. B., Bieda, K., Alibali, M. W., Nathan, M. J., \& Knuth, E. J. (2006). Don't just tell them, show them! Teachers can intentionally alter their instructional gestures. In Proceedings of the 28th Annual Conference of the Cognitive Science Society (pp. 1523-1528). Mahwah, NJ: Erlbaum. doi: 10.1111/j.1467-8624.2007.01027.x

Kline, M. A. (2014). How to learn about teaching: An evolutionary framework for the study of teaching behavior in humans and other animals. Behavioral and Brain Sciences, 1-70. DOI:10.1017/S0140525X14000090.

Macedonia, M., Müller, K., \& Friederici, A. D. (2011). The impact of iconic gestures on foreign language word learning and its neural substrate. $\mathrm{Hu}$ man Brain Mapping, 32, 982-998. DOI:10.1002/hbm.21084.

Marstaller, L., \& Buríanová, H. (2013). Individual differences in the gesture effect on working memory. Psychonomic Bulletin \& Review, 20, 496-500. DOI:10.3758/s13423-012-0365-0.

Novack, M. A., Congdon, E. L., Hemani-Lopez, N., \& Goldin-Meadow, S. (2014). From action to abstraction using the hands to learn math. Psychological Science, 25, 903-910. DOI:10.1177/0956797613518351.

Novack, M., \& Goldin-Meadow, S. (2015). Learning from gesture: How our hands change our minds. Educational Psychology Review, 27, 405-412. DOI:10.1007/s10648-015-9325-3.

Özyürek, A. (2014). Hearing and seeing meaning in speech and gesture: Insights from brain and behaviour. Philosophical Transactions of the Royal Society of London, Series B: Biological Sciences, 36920130296. DOI:10.1098/rstb.2013.0296.

Ping, R., \& Goldin-Meadow, S. (2008). Hands in the air: Using ungrounded iconic gestures to teach children conservation of quantity. Developmental Psychology, 44, 1277-1287. DOI:10.1037/0012-1649.44.5.1277.

Pouw, W. T. J. L., De Nooijer, J. A., Van Gog, T., Zwaan, R. A., \& Paas, F. (2014). Toward a more embedded/extended perspective on the cognitive function of gestures. Frontiers in Psychology, 5, 359. DOI:10.3389/ fpsyg.2014.00359.

Pouw, W., \& Hostetter, A. (in press). Gesture as predictive action. Reti, Saperi, Linguaggi: Italian Journal of Cognitive Sciences.

Pouw, W. T. J. L., Mavilidi, M., Van Gog, T., \& Paas, F. (2016). Gesturing during mental problem solving reduces eye movements, especially for 
individuals with lower visual working memory capacity. Cognitive Processing, 1-9. DOI:10.1007/s10339-016-0757-6.

Smithson, L., \& Nicoladis, E. (2014). Lending a hand to imagery? The impact of visuospatial working memory interference upon iconic gesture production in a narrative task. Journal of Nonverbal Behavior, 38, 247-258. DOI:10.1007/s10919-014-0176-2.

So, W. C., Shum, P. L. C., \& Wong, M. K. Y. (2015). Gesture is more effective than spatial language in encoding spatial information. The Quarterly Journal of Experimental Psychology, 68, 2384-2401. DOI:10.1080/17470218.2015.1015431.

So, W. C., Ching, T. H., Lim, P. E., Cheng, X., \& Ip, K. Y. (2014). Producing gestures facilitates route learning. PLOS ONE, 9, 1-21. DOI:10.1371/ journal.pone.0112543.

Streeck, J., Goodwin, C., \& LeBaron, C. (2011). Embodied interaction: Language and body in the material world. Cambridge: Cambridge University Press.

Valenzeno, L., Alibali, M. W., \& Klatzky, R. (2003). Teachers' gestures facilitate students' learning: A lesson in symmetry. Contemporary Educational Psychology, 28, 187-204. DOI:10.1016/S0361-476X(02) 00007-3.

Van de Weijer-Bergsma, E., Kroesbergen, E. H., Jolani, S., \& van Luit, J. E. H. (2015). The Monkey game: A computerized verbal working memory task for self-reliant administration in primary school children. Behavioral Research Methods. Advance online publication. DOI:10.3758/s13428015-0607-y.

Van de Weijer-Bergsma, E., Kroesbergen, E. H., Prast, E. J., \& Van Luit, J. E. H. (2014). Validity and reliability of an online visual-spatial working memory task for self-reliant administration in school-aged children. Behavioral Research Methods, 47, 708-719. DOI:10.3758/s13428-0140469-8.

Van der Ven, S. H. G., Van der Maas, H. L. J., Straatemeier, M., \& Jansen, B. R. J. (2013). Visuospatial working memory and mathematical ability at different ages throughout primary school. Learning and Individual Differences, 27, 182-192. DOI:10.1016/j. lindif.2013.09.003.

Wagner, S. M., Nusbaum, H., \& Goldin-Meadow, S. (2004). Probing the mental representation of gesture: Is handwaving spatial? Journal of Memory and Language, 50(4), 395-407. DOI:10.1016/j. jml.2004.01.002.

Wechsler, D. (1939). The measurement of adult intelligence. Baltimore, MD: Williams \& Wilkins.

Wu, Y. C., \& Coulson, S. (2014). Co-speech iconic gestures and visuo-spatial working memory. Acta Psychologica, 153, 39-50. DOI:10.1016/j. actpsy.2014.09.002.

\section{APPENDIX A}

Transcript of the route in English including an image of the final route

Translation in English (note though that the explanation was presented in Dutch to the children):

Start at the green dot and walk eastward. At the first crossing go northward, and then move eastward at the second intersection, into the Hammer Street. Then you take the first road northward. At the first intersection go eastward. At the crossroads you take the way northward, into the Guitar Street. At the next crossing you take the street to the west. Go south at the first intersection, into the Tennis Street. Then take the first road westward again. Afterwards go northward, then take the second road westward, into the Painting Street. Immediately go southward. Finally, take the first road westward, until you reach the red dot.

\section{APPENDIX B}

Representation and explanation of the depictive gestures used in the depictive gesture conditions.

Hammer Street: The model moved her right arm up and down as if hitting a nail with a hammer (Figure B1a).

Guitar Street: The model held her left arm as if holding a guitar and moved her right arm up and down as if playing the strings (Figure B1b).

Tennis Street: The video used her left arm as if throwing a ball up in the air and then used her right arm as if hitting the ball with a racket (Figure B1c).

Painting Street: The video model moved her right arm up and down as if using a paint brush to paint (Figure B1d). a

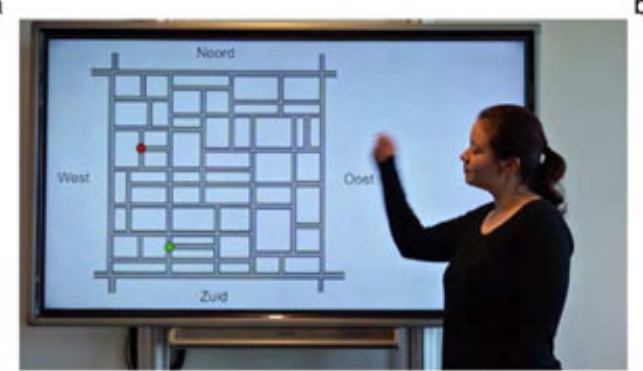

c

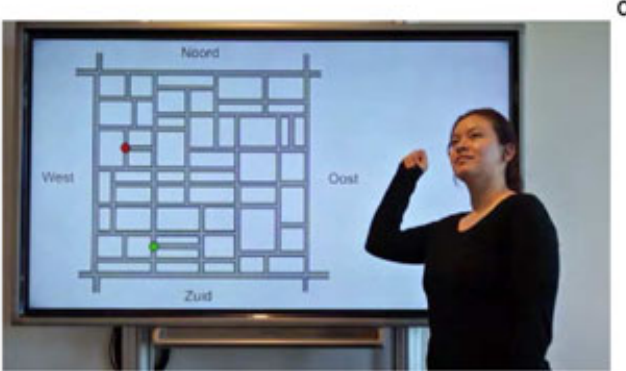

b

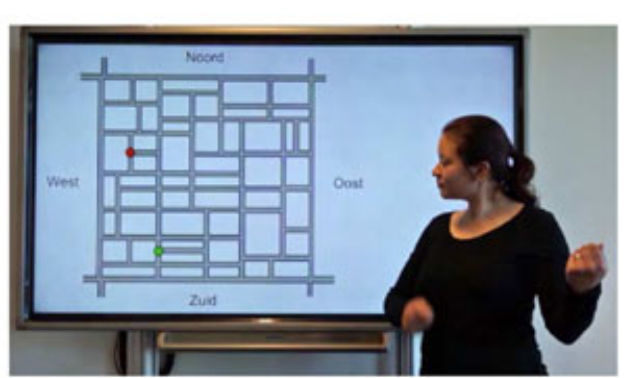

d

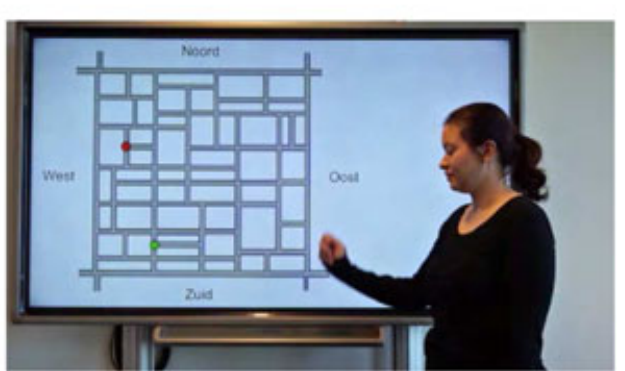

Figure B1. Depictive gesture for a) 'Hammer Street'; b) 'Guitar Street'; c) 'Tennis Street'; and d) 'Painting Street'. 